Claremont Colleges

Scholarship@ Claremont

All HMC Faculty Publications and Research

HMC Faculty Scholarship

$12-1-1994$

\title{
Portable, High-Bandwidth Frequency-Domain Photon Migration Instrument for Tissue Spectroscopy
}

Steen J. Madsen

University of Nevada, Las Vegas

Eric R. Anderson

University of California - Irvine

Richard C. Haskell

Harvey Mudd College

Bruce J. Tromberg

University of California - Irvine

\section{Recommended Citation}

Madsen, SJ, Anderson, ER, Haskell, RC, Tromberg, BJ. Portable, high-bandwidth frequency-domain photon migration instrument for tissue spectroscopy. Opt Lett. 1994;19(23): 1934-1936.

This Article is brought to you for free and open access by the HMC Faculty Scholarship at Scholarship @ Claremont. It has been accepted for inclusion in All HMC Faculty Publications and Research by an authorized administrator of Scholarship @ Claremont. For more information, please contact scholarship@cuc.claremont.edu. 


\title{
Portable, high-bandwidth frequency-domain photon migration instrument for tissue spectroscopy
}

\author{
Steen J. Madsen, Eric R. Anderson, Richard C. Haskell, and Bruce J. Tromberg \\ Beckman Laser Institute and Medical Clinic, University of California, Irvine, Irvine, California 92715
}

Received July 5, 1994

\begin{abstract}
We describe a novel frequency-domain photon migration instrument employing dírect diode laser modulation and avalanche photodiode detection, which is capable of noninvasively determinating the optical properties of biological tissues in near real time. An infinite medium diffusion model was used to extract absorption and transport scattering coefficients from $300-\mathrm{kHz}$ to $800-\mathrm{MHz}$ photon-density wave phase data. Optical properties measured in tissue-simulating solutions at $670 \mathrm{~nm}$ agreed to within $10 \%$ of those expected.
\end{abstract}

Knowledge of the optical properties of biological tissue is essential in both diagnostic and therapeutic applications of lasers in medicine. To be useful clinically, the optical properties should be determined quickly and noninvasively, which may be possible by use of frequency-domain photon migration (FDPM).

In FDPM the intensity of light incident upon a material, such as biological tissue, is modulated at a high frequency $\left(10^{7}-10^{9} \mathrm{~Hz}\right.$ ), and the diffusely reflected or transmitted signal is measured by a phase-sensitive detector. It has been demonstrated that intensity-modulated light propagates through multiple-scattering media (e.g., biological tissue) as diffuse photon density waves with a coherent front. ${ }^{1-3}$ As density wave dispersion is a function of the optical properties of the material probed, one can determine absorption $\left(\mu_{a}\right)$ and transport scattering $\left(\mu_{s}^{\prime}\right)$ coefficients by fitting the measured frequency- and distance-dependent behavior to analytical expressions derived from diffusion theory. ${ }^{3}$

In contrast to more sensitive time-domain photon propagation techniques, such as time-correlated single-photon counting, ${ }^{4}$ FDPM signals are continuous and can generally be acquired more rapidly and economically. Thus FDPM methods seem to be more suitable for portable, real-time, noninvasive physiological monitoring.

Several modulation-detection techniques have been developed for FDPM. The most general consists of external Pockels cell modulation of the light source (arc lamp or laser) and cross-correlation detection by use of conventional photomultiplier tubes. ${ }^{5}$ However, because of its limited bandwidth $(200-300 \mathrm{MHz})$, Pockels cell-photomultiplier tube instrumentation may be insufficient for characterizing optical properties of many biological tissues. For example, FDPM measurements on human uterine tissue indicate that frequencies in excess of $350 \mathrm{MHz}$ are required for reliable determination of $\mu_{a}$ and $\mu_{s}^{\prime}$ in a single measurement. ${ }^{6}$ This bandwidth limitation can be overcome by use of the high harmonic content of pulsed mode-locked lasers and fast photodetectors such as microchannel plate photomultiplier tubes.
Although such systems are ill suited to use in the clinic because of their cost and complexity, they permit recording of photon-density wave dispersion with sufficient temporal resolution for optical properties to be calculated in a single measurement.

In this Letter we describe a relatively simple, inexpensive diode-based FDPM system that operates in near real time between $300 \mathrm{kHz}$ and $1 \mathrm{GHz}$. This FDPM system is unique because we employ, for the first time to our knowledge, direct diode laser modulation and avalanche photodiode detection. As a result, we can rapidly and reliably calculate optical properties in a single multifrequency measurement.

A schematic overview of the system is illustrated in Fig. 1. One of the unique features of the system is the vector network analyzer (Hewlett-Packard Model 8753C), which measures reflection and transmission characteristics of devices and networks by applying a known swept signal and measuring the responses. Such a device has been used to measure electrical properties of biological tissues. ${ }^{7}$ The analyzer's built-in synthesized source produces a swept rf signal ( $300 \mathrm{kHz}$ to $1 \mathrm{GHz}$ ), which is superimposed upon the direct current of a GaAs $(\lambda=670 \mathrm{~nm})$ laser diode (SDL, Inc., Model 7420) by means of a bias tee (Picosecond Pulse Labs Model 5575A). The direct current is obtained from a stabilized current source (ILX Lightwave Model LDC-3742), which also provides thermoelectric cooling control for the laser diode. The laser was biased $25 \mathrm{~mA}$ above lasing threshold ( $200 \mathrm{~mA})$, and $20 \mathrm{dBm}$ of $\mathrm{rf}$ power was applied. Since no impedance matching of the diode to the $50-\Omega$ source impedance was performed, the actual ac optical power was much less than $20 \mathrm{dBm}$. Although slight distortion of the sinusoidal output of the laser could be observed at some modulation frequencies, we could reduce it by changing the dc bias on the laser diode. The laser output was coupled directly into a $600-\mu \mathrm{m}$ step-index multimode fiber, which was incident upon the material. The average optical power coupled into the sample ranged from 30 to $40 \mathrm{~mW}$. Diffusely propagating photon density waves were col- 


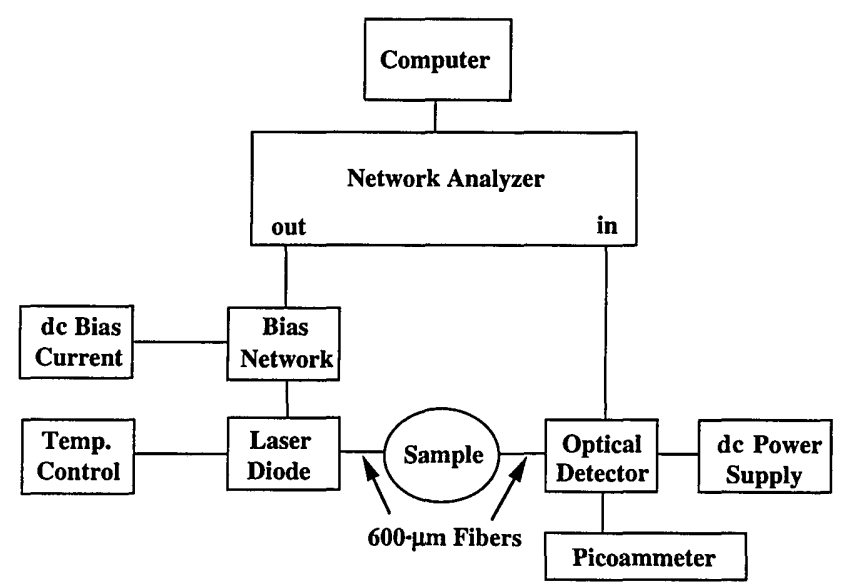

Fig. 1. Schematic of the frequency-domain instrument.

lected by an identical fiber and transmitted to a high-gain (approximately $4 \times 10^{3}$ ) avalanche photodiode (APD; New Focus, Inc., Model 1651) biased at $15 \mathrm{~V}$ (New Focus, Inc., dc power supply \#0901). The dc output of the APD was monitored with a picoammeter (Keithley Model 485). Acquisition times of photon-density-wave phase and amplitude over the entire $300-\mathrm{kHz}$ to $1-\mathrm{GHz}$ frequency spectrum are variable and depend primarily on intermediate frequency bandwidth and signal averaging parameters. Reasonable signals can be acquired on the order of seconds. Shorter acquisition times can also be realized by constraining the measurement bandwidth.

The source modulation at $500 \mathrm{MHz}$ is illustrated in Fig. 2 for a $225-\mathrm{mA}$ dc bias setting. There is some distortion of the sinusoidal waveform (particularly near threshold), which is due to the dynamic behavior of the semiconductor laser under strong modulation. ${ }^{8}$ By adjusting the dc bias to $250 \mathrm{~mA}$ we were able to reduce this distortion at some expense to overall source modulation depth. As a result, dc bias was maintained at $225 \mathrm{~mA}$ for all subsequent experiments. Although the $500-\mathrm{MHz}$ response is the only waveform shown, modulation quality generally improves at lower frequencies and degrades with increasing frequency. In practical terms, we were able to compensate for small waveform distortions by acquiring sample and reference data under identical conditions (see below).

The ability of the system to determine the optical properties of tissue-simulating solutions was tested. The solutions consisted of $2 \%$ Intralipid (Kabivitrum, Inc., Clayton, N.C.) and various concentrations of nickel tetrasulfonated phthalocyanine (NiSPC; Midcentury, Posen, Ill.). Intralipid is a highly scattering and low absorbing phospholipid emulsion with well-characterized optical properties, and NiSPC is a strong absorber at $670 \mathrm{~nm}$. The concentrations of Intralipid and NiSPC were chosen to correspond to the optical properties of typical tissues. In all cases the source and detector fibers were centered and placed at a depth of approximately $10 \mathrm{~cm}$ in a 4-L cylindrical beaker containing $3.5 \mathrm{~L}$ of the liquid solution. This corresponds to an infinite geometry. The infinite geometry solution of the diffusion equation is straightforward and, in terms of the phase lag $(\phi)$, is given by ${ }^{3}$

$$
\begin{aligned}
\phi=r\left[\mu_{a}\left(\mu_{a}+\mu_{s}^{\prime}\right)\right]^{1 / 2}\left(\frac{2}{3}\right)^{1 / 2} & \\
& \times\left\{\left[1+\left(\frac{\omega}{\mu_{a} c}\right)^{2}\right]^{1 / 2}-1\right\}^{1 / 2} .
\end{aligned}
$$

A nonlinear least-squares fitting routine, based on a Marquardt-Levenberg algorithm, was used to fit the diffusion expression [Eq. (1)] to the experimental data. A reference measurement was taken with both source and detector fibers inserted in the solution and separated by $4 \mathrm{~mm}$. The results are illustrated in Fig. 3.

Figure 3 shows phase versus frequency plots as a function of added absorber concentration at a source-detector separation of $14 \mathrm{~mm}$ (effective separation of $10 \mathrm{~mm}$ because the reference was recorded at a separation of $4 \mathrm{~mm}$ ). Each data set was acquired in approximately $14 \mathrm{~s}$. As expected, the phase decreases with increased absorber, as longerpath-length photons have a greater probability of

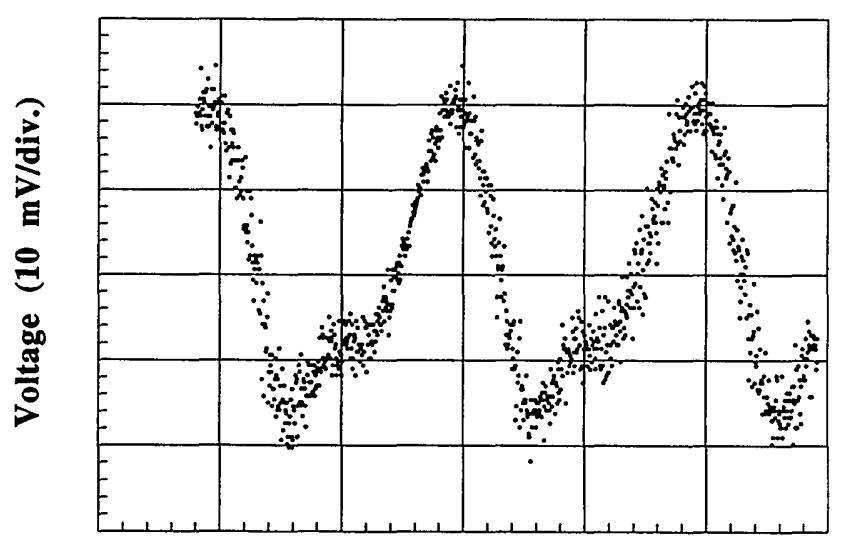

Time (100 ns/div.)

Fig. 2. Source modulation at $500 \mathrm{MHz}$ and dc bias of $225 \mathrm{~mA}$.

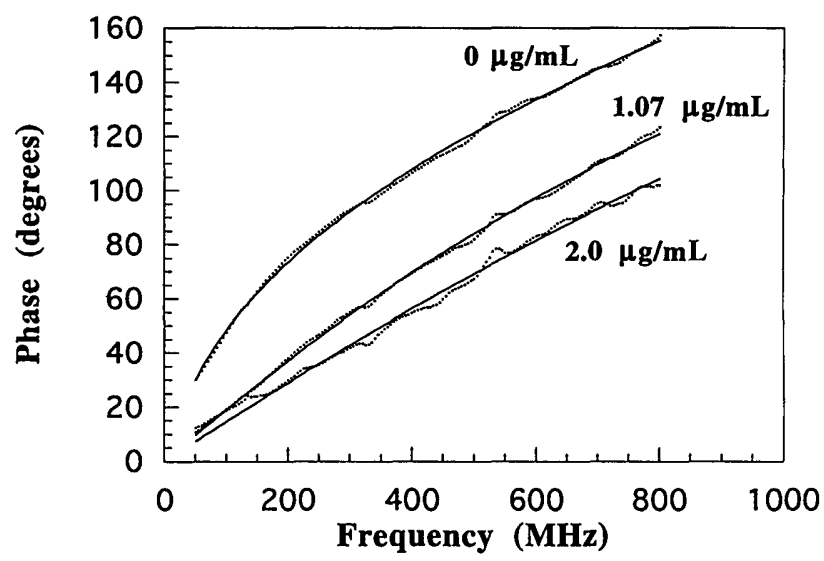

Fig. 3. Phase versus frequency response for $2 \%$ Intralipid as a function of NiSPC concentration. The curves represent beat fits to the data. 
Table 1. Summary of Fitted and Actual Optical Properties

\begin{tabular}{ccccc}
\hline $\begin{array}{c}\mathrm{NiSPC} \\
(\mu \mathrm{g} / \mathrm{mL})\end{array}$ & $\begin{array}{c}\mu_{a \text { fit }} \\
\left(\mathrm{mm}^{-1}\right)\end{array}$ & $\begin{array}{c}\mu_{a \text { actual }} \\
\left(\mathrm{mm}^{-1}\right)\end{array}$ & $\begin{array}{c}\mu_{s^{\prime} \text { fit }} \\
\left(\mathrm{mm}^{-1}\right)\end{array}$ & $\begin{array}{r}\mu_{s^{\prime} \text { actual }} \\
\left(\mathrm{mm}^{-1}\right)\end{array}$ \\
\hline 0 & $(8.52 \pm 0.51) \times 10^{-4}$ & $8.52 \times 10^{-4}$ & $2.28 \pm 0.14$ & 2.42 \\
1.07 & $(1.18 \pm 0.07) \times 10^{-2}$ & $1.24 \times 10^{-2}$ & $2.22 \pm 0.14$ & 2.42 \\
2.0 & $(2.01 \pm 0.12) \times 10^{-2}$ & $2.16 \times 10^{-2}$ & $2.22 \pm 0.14$ & 2.42 \\
\hline
\end{tabular}

being absorbed. It is shown that, even with the addition of the absorber, reliable phase data are obtained up to $800 \mathrm{MHz}$. The actual and fitted optical properties are summarized in Table 1. In all cases the transport scattering coefficient agrees to within $10 \%$ of that predicted by Mie theory ( $\mu_{s^{\prime} \text { actual }}$ in Table 1$){ }^{9}$ Significant variations in the absorption coefficient of Intralipid have been reported in the literature. ${ }^{10}$ These differences are probably due to inconsistencies in the manufacturing process. For that reason the actual absorption coefficient for pure Intralipid in Table 1 is taken to be that obtained from the fit. This value is well within the range of that found by others. ${ }^{10}$ In all cases the fitted absorption coefficient is in excellent agreement with the actual value based on the absorption of Intralipid and the absorption of $1 \mu \mathrm{g} / \mathrm{mL} \mathrm{NiSPC}\left(1.08 \times 10^{-2} \mathrm{~mm}^{-1}\right)$. The uncertainties quoted in Table $1(6 \%)$ are due mainly to uncertainties in the source-detector separation.

We examined phase precision by repeating measurements at a given source-detector separation. The results of 10 such measurements revealed a phase precision of between $0.5 \%$ and $1 \%$. We evaluated long-term phase stability by recording the phase at 5-min intervals over a period of 30 min under identical conditions. The phase was found to be stable to within $2 \%$ at frequencies above $100 \mathrm{MHz}$. Below $100 \mathrm{MHz}$ the phase stability was rather poor $(5-10 \%)$.

In general, we were able to obtain reliable phase information up to $800 \mathrm{MHz}$ at source-detector separations up to $18 \mathrm{~mm}$ in the $1.07-\mu \mathrm{g} / \mathrm{mL}$ tissuesimulating solution. Rapid attenuation of the ac signal component limited the bandwidth of our measurements at greater distances. For example, at a source-detector separation of $20 \mathrm{~mm}$, phase measurements were limited to frequencies below $450 \mathrm{MHz}$. In its current configuration the overall system bandwidth is detector limited. The APD has a 3-dB rolloff at $1 \mathrm{GHz}$; however, the response of the detector falls by $1.5 \mathrm{~dB}$ at $800 \mathrm{MHz}$ at the high-gain settings used in these experiments.

Modifications to the present system should improve signal-to-noise ratio characteristics and permit reliable measurements up to the $1-\mathrm{GHz}$ APD limit. Such modifications ratio include (1) improving the optical coupling onto the small-area (200$\mu \mathrm{m}$-diameter) APD detector, (2) enhancing overall detector gain by electronic amplification of the APD signal, (3) improving source modulation characteristics by placing a low-inductance resistor in series with the laser diode so the overall impedance is matched to the $50-\Omega$ rf output, (4) increasing the source modulation depth by amplifying the network analyzer rf output, and (5) combining phase data with the high-quality ac and dc amplitude information (data not shown) to calculate optical properties.

In summary, we describe the development of a high-bandwidth FDPM instrument, which, for the first time to our knowledge, employs direct diode modulation and APD detection at multiple modulation frequencies up to $1 \mathrm{GHz}$. Access to $1-\mathrm{GHz}$ multifrequency phase and amplitude information permits rapid, noninvasive characterization of the optical properties of most commonly encountered tissues in a single measurement. With the incorporation of additional wavelength diodes the system will be capable of monitoring various physiological parameters, including oxygenated and deoxygenated hemoglobin levels, drug concentration, blood volume changes, tissue hydration status, and tissue scattering properties. As the instrument is compact, it can easily be transported to operating rooms and bedridden patients, such as neonates in intensive care. These features suggest that portable FPDM devices can be constructed to provide high-bandwidth information for a variety of applications in tissue diagnostics and imaging.

This research was supported by the Whitaker Foundation (WF16493), the National Institutes of Health (R29GM50958), and Beckman Instruments, Inc. Address any correspondence to B. J. Tromberg.

\section{References}

1. J. Fishkin, E. Gratton, M. J. vandeVen, and W. W. Mantulin, Proc. Soc. Photo-Opt. Instrum. Eng. 1431, 122 (1991).

2. M. A. O'Leary, D. A. Boas, B. Chance, and A. G. Yodh, Phys. Rev. Lett. 69, 2658 (1992).

3. B. J. Tromberg, L. O. Svaasand, T.-T. Tsay, and R. C. Haskell, Appl. Opt. 32, 607 (1993).

4. D. V. O'Conner and D. Phillips, Time-Correlated Single Photon Counting (Academic, New York, 1984), Chap. 1, pp. 9-34.

5. J. R. Lakowicz, Principles of Fluorescence Spectroscopy (Plenum, New York, 1983), Chap. 3, pp. 51-91.

6. S. J. Madsen, P. Wyss, L. O. Svaasand, R. C. Haskell, Y. Tadir, and B. J. Tromberg, Phys. Med. Biol. 39, 1191 (1994).

7. W. T. Joines, Y. Zhang, C. Li, and R. L. Jirtle, Med. Phys. 21, 547 (1994).

8. W. I. Way, J. Lightwave Technol. LT-5, 305 (1987).

9. H. J. van Staveren, C. J. M. Moes, J. van Marle, S. A. Prahl, and M. J. C. van Gemert, Appl. Opt. 30, 4507 (1991).

10. S. T. Flock, S. L. Jacques, B. C. Wilson, W. Star, and M. J. C. van Gemert, Lasers Surg. Med. 12, 510 (1992). 